\title{
Transcriptomic analysis of flower opening response to relatively low temperatures in Osmanthus fragrans
}

Jianxin $\mathrm{Fu}^{\dagger}$, Chao Zhang ${ }^{\dagger}$, Yucheng Liu, Tianhong Pang, Bin Dong, Xiaoyue Gao, Yimin Zhu and Hongbo Zhao*

\begin{abstract}
Background: Sweet osmanthus (Osmanthus fragrans Lour.) is one of the top ten traditional ornamental flowers in China. The flowering time of once-flowering cultivars in $O$. fragrans is greatly affected by the relatively low temperature, but there are few reports on its molecular mechanism to date. A hypothesis had been raised that genes related with flower opening might be up-regulated in response to relatively low temperature in $O$. fragrans. Thus, our work was aimed to explore the underlying molecular mechanism of flower opening regulated by relatively low temperature in $O$. fragrans.

Results: The cell size of adaxial and abaxial petal epidermal cells and ultrastructural morphology of petal cells at different developmental stages were observed. The cell size of adaxial and abaxial petal epidermal cells increased gradually with the process of flower opening. Then the transcriptomic sequencing was employed to analyze the differentially expressed genes (DEGs) under different number of days' treatments with relatively low temperatures $\left(19^{\circ} \mathrm{C}\right)$ or $23^{\circ} \mathrm{C}$. Analysis of DEGs in Gene Ontology analysis showed that "metabolic process", "cellular process", "binding", "catalytic activity", "cell", "cell part", "membrane", "membrane part", "single-organism process", and "organelle" were highly enriched. In KEGG analysis, "metabolic pathways", "biosynthesis of secondary metabolites", "plant-pathogen interaction", "starch and sucrose metabolism", and "plant hormone signal transduction" were the top five pathways containing the greatest number of DEGs. The DEGs involved in cell wall metabolism, phytohormone signal transduction pathways, and eight kinds of transcription factors were analyzed in depth.

Conclusions: Several unigenes involved in cell wall metabolism, phytohormone signal transduction pathway, and transcription factors with highly variable expression levels between different temperature treatments may be involved in petal cell expansion during flower opening process in response to the relatively low temperature. These results could improve our understanding of the molecular mechanism of relatively-low-temperature-regulated flower opening of $O$. fragrans, provide practical information for the prediction and regulation of flowering time in $O$. fragrans, and ultimately pave the way for genetic modification in $O$. fragrans.
\end{abstract}

Keywords: Flower development, Petal epidermal cells, Temperature, Sweet osmanthus, RNA-seq analysis

\footnotetext{
* Correspondence: zhaohb@zafu.edu.cn

${ }^{\dagger}$ Jianxin Fu and Chao Zhang contributed equally to this work.

Zhejiang Provincial Key Laboratory of Germplasm Innovation and Utilization

for Garden Plants, Key Laboratory of National Forestry and Grassland

Administration on Germplasm Innovation and Utilization for Southern

Garden Plants, School of Landscape Architecture, Zhejiang Agriculture and

Forestry University, Hangzhou 311300, Zhejiang, P.R. China
}

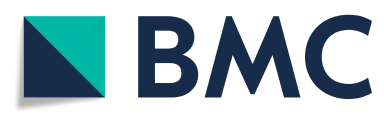

(c) The Author(s). 2020 Open Access This article is licensed under a Creative Commons Attribution 4.0 International License, which permits use, sharing, adaptation, distribution and reproduction in any medium or format, as long as you give appropriate credit to the original author(s) and the source, provide a link to the Creative Commons licence, and indicate if changes were made. The images or other third party material in this article are included in the article's Creative Commons licence, unless indicated otherwise in a credit line to the material. If material is not included in the article's Creative Commons licence and your intended use is not permitted by statutory regulation or exceeds the permitted use, you will need to obtain permission directly from the copyright holder. To view a copy of this licence, visit http://creativecommons.org/licenses/by/4.0/. The Creative Commons Public Domain Dedication waiver (http://creativecommons.org/publicdomain/zero/1.0/) applies to the data made available in this article, unless otherwise stated in a credit line to the data. 


\section{Background}

Osmanthus fragrans Lour. (Oleaceae) is one of the top ten traditional ornamental plants in China with more than 2500 years' history of cultivation [1]. It is a small evergreen tree, grown as ornamental plants for its fragrant edible flowers. Cultivars of $O$. fragrans could be divided into once-flowering group and recurrent-flowering group according to different flowering habits [2]. The once-flowering cultivars bloom in autumn and the flowering time varies greatly in different areas, such as in Hangzhou, Shanghai, Nanjing, and Suzhou, or even in different years in the same area [3]. The researches on the flowering time of different cultivars indicated that relatively low temperature before blooming is the most important environmental factor determining the flower opening of $O$. fragrans $[3,4]$. However, the knowledge of molecular mechanism of flower opening in O. fragrans, especially response to relatively low temperature, still remains limited.

In many higher plants, the growth of flower petals is the most remarkable process during flower opening. Flower petals are the most important component of reproductive organs and play vital roles in attracting the suitable pollinator(s). Flower color, size, shape and appearance, which are determined by flower petal, are important traits appreciated by the breeders and consumers [5]. One of the traits, the size of flower petal is determined by cell division at early phases of petal growth and cell expansion at later stages of flower opening [6].

Cell expansion is accompanied by a series of process including cell wall loosening, cellulose biosynthesis, polysaccharides conversion into soluble carbohydrate, ion and water uptake, and cytoskeleton rearrangement [7]. Cell wall loosening proteins include expansin (EXP), xyloglucan endotransglycosylase/hydrolase $(\mathrm{XTH})$, endo-1,4- $\beta$-D-glucanase, and pectinase [8]. Among them, EXP and XTH particularly participate in disrupting the non-covalent bonds between the cellulose microfibrils and the crosslinking glycans of the cell wall to increase the cellwall creep rate, while pectinase and endo-1,4- $\beta$-D-glucanase can degrade the cell wall [9]. In wintersweet (Chimonanthus praecox), the expressions of EXP genes increase during flower opening [10]. The developing petals of carnation show high activities of cellulase and pectin esterase [11]. These findings reveal that petal growth relevant to flower opening is probably attributed to cell expansion. Moreover, soluble carbohydrates depending on the degradation of polysaccharides can act as osmotically active compounds which could lower the osmotic water potential and facilitate water influx in order to allow cell expansion [12]. The concentration of soluble carbohydrates in the petals will increase in the flower opening process of plants such as carnation [13], rose [14], chrysanthemum [15], Tweedia caerulea [16], and lisianthus [17].

Cell expansion is regulated by both external factors, such as temperature, humidity, and the quality and quantity of light, and internal factors, such as the circadian clock and phytohormones [18, 19]. Phytohormones are the most important mediators regulating flower opening and could be affected by circadian factors or environmental factors. As far as the present researches are concerned, most of the phytohormones, such as auxin (AUX), gibberellin (GA), ethylene (ETH), brassinosteroid (BR), jasmonic acid (JA) and abscisic acid (ABA), are proven to affect flower opening [5, 6, 20-22].

In this research, potted plants of $O$. fragrans 'Yanhong Gui' were employed as materials to research the effects of different temperature conditions on flower opening process under controlled relatively low temperatures. Then the transcriptomic sequencing was used to analyze the differentially expressed genes (DEGs) after different number of days' treatment under relatively high or low temperatures to figure out the key genes involved in the regulation of flower opening in relation to relatively low temperature. This study would lay foundation on fully revealing the molecular mechanism of relatively-lowtemperature-regulated flower opening of $O$. fragrans and provide theoretical reference for the prediction and regulation of flowering time and genetic modification in O. fragrans.

\section{Results}

Scanning electron microscopy (SEM) and transmission electron microscopy (TEM) analysis

Developmental stages of sweet osmanthus flowers for SEM and TEM analysis were described as follows: stage 1 (S1), the outer bud scales unfurled and the inner bud scales still furled; S2, the bud became globular-shaped and the inside bracts covering the inflorescence was visible; S3, the inflorescence burst through bracts and the florets closely crowded; S4, initial flowering stage; S5, full flowering stage; S6, pollen-scattered stage. Observation on the cell size of adaxial and abaxial petal epidermal cells with SEM revealed that cell size of adaxial petal epidermal cells increased gradually $(P<0.05$, by Duncan's multiple range test) from S1 to S6 (Fig. 1a, Table S1), while that of abaxial petal epidermal cells enlarged gradually from S1 to S5, reached the peak at S5, and then greatly decreased from S5 to S6 (Fig. 1b, Table $\mathrm{S} 1$ ). These results were coincident with results in Gaillardia grandiflora [23], carnation [24] and T. caerulea [16], indicating that the cell division ceased and cell expansion occurred during floral opening process. However, in rose [25] and E. grandiflorum [26] cell division and cell expansion simultaneously appeared during this 

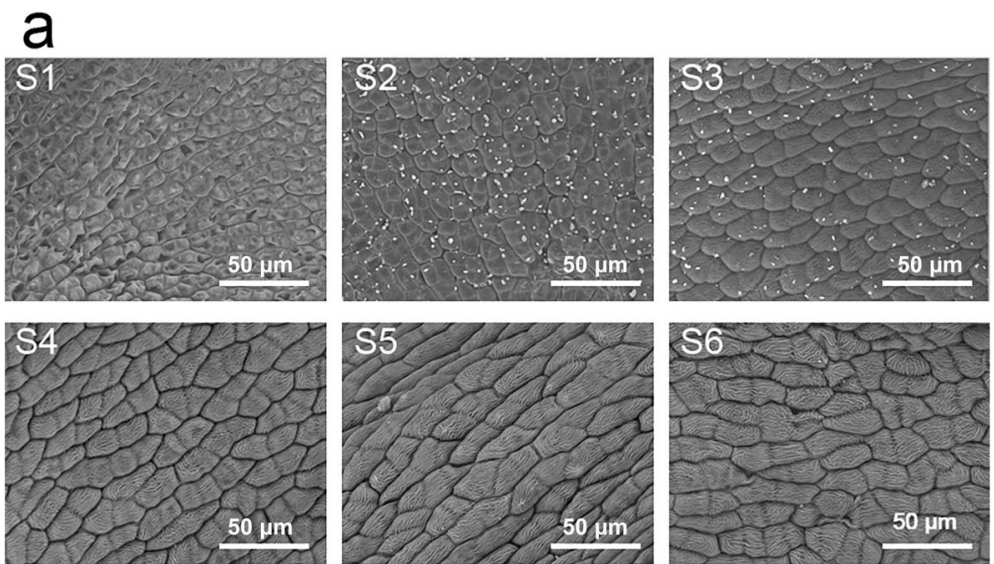

\section{b}
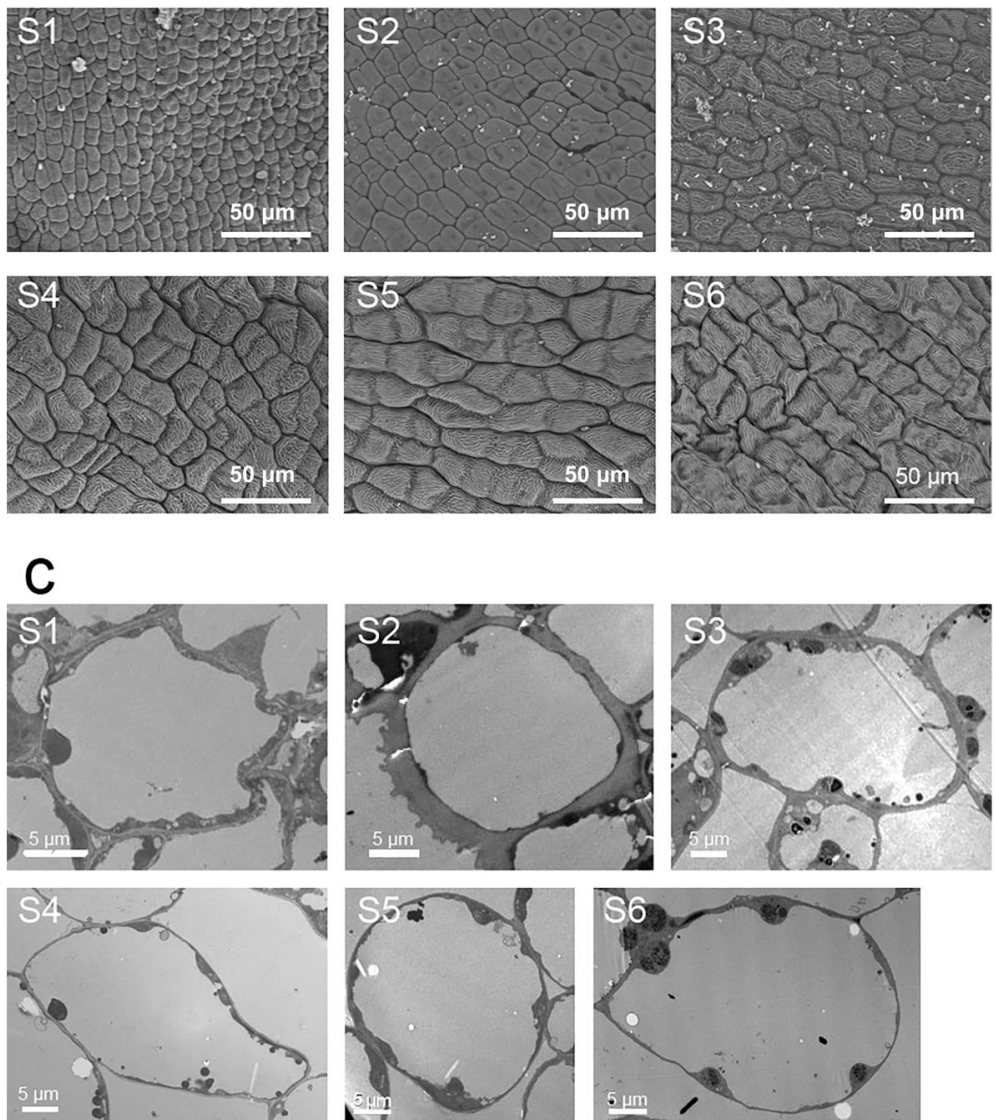

Fig. 1 Observation on the petal cells of sweet osmanthus flower from S1 to S6 with SEM and TEM. a Observation on adaxial petal epidermal cells with SEM. White bar $50 \mu \mathrm{m}$; b Observation on abaxial petal epidermal cells with SEM. White bar $50 \mu \mathrm{m}$; c Observation on adaxial petal epidermal cells with TEM. White bar $5 \mu \mathrm{m}$. S1), the outer bud scales unfurled and the inner bud scales still furled; S2, the bud became globular-shaped and the inside bracts covering the inflorescence was visible; $\$ 3$, the inflorescence burst through bracts and the florets closely crowded; $\$ 4$, initial flowering stage; S5, full flowering stage; S6, pollen-scattered stage

process. What's more, based on TEM observation, it was found that vacuole occupied most area of adaxial petal epidermal cells in O. fragrans (Fig. 1c, Table S1). The same situation occurred in E. grandiflorum [26]. These results indicated that the petal cell expansion was accompanied by the enlargement of vacuole. Vacuole size increased gradually from S1 to S6. At S2, vacuole size of cells was nearly two times as large as that at S1. With the cell development, vacuole size of cells at S6 was just one time larger than that at S2 (Fig. 1c, Table 
S1), suggesting that the growth rate from S1 to S2 was the highest during flower development.

\section{Bud appearance with treatments}

Before $23^{\circ} \mathrm{C}$ or $19^{\circ} \mathrm{C}$ treatment, the buds of all plants were at $\mathrm{S} 1$, and they were spindle-shaped, with unfurled outer bud scales and furled inner bud scales. Bud appearance remained the same under $23^{\circ} \mathrm{C}$ treatment for 2 $\mathrm{d}, 4 \mathrm{~d}$, and $6 \mathrm{~d}(\mathrm{H} 2, \mathrm{H} 4, \mathrm{H} 6)$, as well as the $19^{\circ} \mathrm{C}$ treatment for $2 \mathrm{~d}$ and $4 \mathrm{~d}$ (L2 and L4). When treated with $19{ }^{\circ} \mathrm{C}$ treatment for $6 \mathrm{~d}$ (L6), the bud stage reached at $\mathrm{S} 2$, suggesting that the relatively low temperature, like $19^{\circ} \mathrm{C}$, helps 'Yanhong Gui' blossom in experimental condition.

\section{Illumina sequencing and de novo assembly of sequence reads}

To reveal the effect of $19{ }^{\circ} \mathrm{C}$ on the transcriptomic change in 'Yanhong Gui', we collected three biological replicate samples of H2, H4, H6, L2, L4, and L6 for reference transcriptome sequencing and RNA-seq analysis. Reference transcriptome sequencing generated 164,753, 984 raw reads and 110,307,366 clean reads (Table S2). A total of 152,247 transcripts with average length of $751 \mathrm{nt}$ were obtained and 96,920 unigenes with average length of $873 \mathrm{nt}$ were yielded after all clean reads were assembled (Table S3).

\section{Functional annotation and classification}

The assembled unigenes were aligned to Non-Redundant Protein Sequence Database (NR), Nucleotide Sequence Database (NT), Swiss-Prot Database, Kyoto Encyclopedia of Genes and Genomes (KEGG) Database, Eukaryotic Orthologous Groups of proteins (KOG) Database, Gene Ontology (GO) Database, and InterPro Database, in order to obtain the putative annotations (Table S4). Totally, 61, $654(63.61 \%)$ unigenes were successfully annotated using at least one database, while 8150 (8.41\%) unigenes were annotated using all seven databases. Total 57,721 (59.56\%) of unigenes were annotated using NR database, $59.26 \%$ of which have high homology to sequences from Sesamum indicum (Fig. S1). In total, 37,284 unigenes were matched in the Swiss-Prot database, which is about $38.47 \%$ of all annotated unigenes (Table S4). There were 40,764 unigenes mapped into 134 KEGG pathways which can be divided into six large pathways including "cellular processes", "environmental information processing", "genetic information processing", "human diseases", "metabolism", and "organismal systems" (Table S5). The pathways with the highest numbers of unigenes were "metabolic pathways" (Ko01100, 8561 unigenes, 21\%), followed by "biosynthesis of secondary metabolites" (Ko01110, 4440 unigenes, 10.89\%), "plant-pathogen interaction" (Ko04626, 1876 unigenes, 4.6\%), and "plant hormone signal transduction" (Ko04075, 1444 unigenes, 3.54\%) (Table S5). Next, GO analysis was performed and a total of 16 , 014 unigenes (16.52\% of all annotated unigenes) were categorized into $53 \mathrm{GO}$ terms under three main categories: biological process, cellular component, and molecular function (Fig. S2). Proteins related to "metabolic process", "cellular process", and "single-organism process" were enriched in biological processes. The "single-organism process" category defines a biological process involving only one organism, and the 4806 unigenes annotated as such may be related to flowering organisms specifically. In the cellular component category, the "cell", "cell part", and "membrane" were the most highly presented GO terms. Under the molecular function category, the "catalytic activity", and "binding proteins" were the most enriched (Fig. S2). We also performed KOG analysis to evaluate the functions of the assembled unigenes and 43,496 unigenes were assigned $25 \mathrm{KOG}$ classification (Fig. S3). Among the 25 KOG classification, the cluster of "general function prediction only" (11,753 unigenes, 27.02\%) represented the largest group, followed by "signal transduction mechanisms" (6000 unigenes, 13.79\%) and "function unknown" (4761, 10.95\%). "Cell motility" has the smallest proportion, only accounted for $0.20 \%$ (Fig. S3). Additionally, 46,405 $(47.88 \%)$ unigenes in the transcriptome library were annotated against InterPro database (Table S4).

RNA-sequencing (RNA-seq) and mapping to the reference transcriptome database

Eighteen cDNA libraries for RNA-seq analysis were respectively sequenced, generating 23.58-24.14 Mb raw reads (Table S2). Furthermore, we obtained 23.58-24.13 $\mathrm{Mb}$ clean reads in $18 \mathrm{cDNA}$ libraries, with the clean data rate of $99.94-99.97 \%$. Then, these clean reads were respectively mapped to the reference transcriptome database. Total mapped reads percentage and unique match percentage in the 18 libraries were similar. Total mapped reads percentage ranged from 86.39 to $88.44 \%$, and unique match percentage ranged from 53.7 to $56.29 \%$ (Table S6). The correlation coefficients among biological replications are greater than 0.94 in all samples (Fig. 2, Table S7).

\section{Screening and analysis of DEGs}

We performed a detailed comparative analysis of the DEGs ( $P$ value $\leq 0.01$ and $\mid \log _{2}$ Ratio $\mid \geq 1$ ) in three comparisons including $\mathrm{L} 2$ vs $\mathrm{H} 2$, L4 vs $\mathrm{H} 4$, and L6 vs H6. In the $\mathrm{L} 2$ vs $\mathrm{H} 2$ comparison, there were 7365 and 15,864 unigenes were down-regulated and up-regulated, respectively. Meanwhile, 8393 and 22,755 unigenes were down-regulated and up-regulated in the L4 vs H4 combination, respectively. In the L6 vs H6 comparison, 9551 unigenes were down-regulated and 22,011 unigenes were up-regulated, respectively (Fig. 3a). In total, there were 


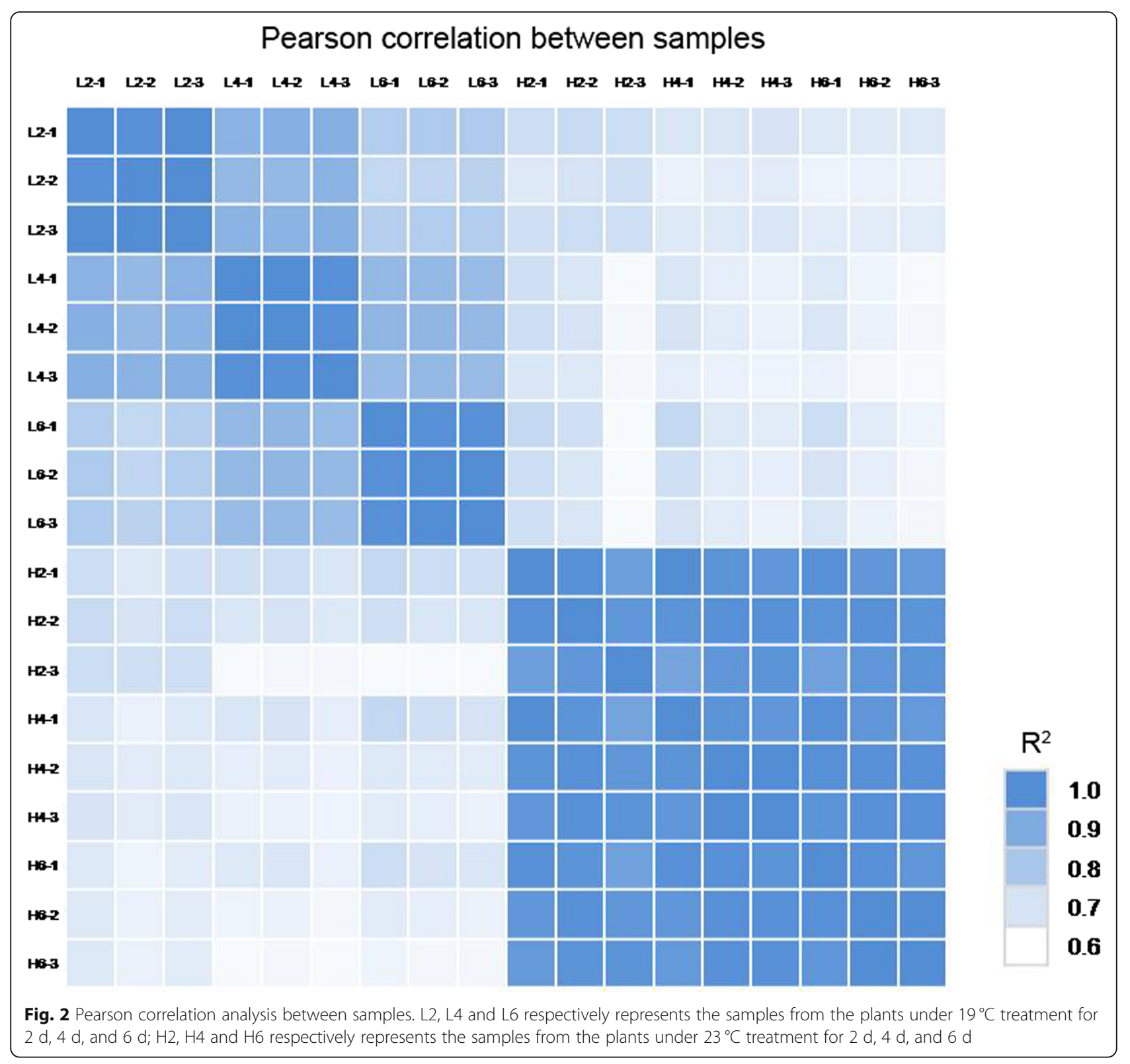

more up-regulated unigenes than down-regulated unigenes during floral opening under relatively low temperature. Additionally, 7496 up-regulated DEGs were shared by three comparisons, while 4108, 6132, and 6913 up-regulated DEGs were only up-regulated in the L2 vs H2, L4 vs H4, and L6 vs H6 comparison, respectively (Fig. 3b). What's more, 2968 down-regulated DEGs were shared by three comparisons, while 2954, 2189, and 3806 down-regulated unigenes were only downexpressed in the L2 vs H2, L4 vs H4, and L6 vs H6 comparison, respectively (Fig. 3c).

\section{GO enrichment and KEGG pathway analysis of DEGs}

In this work, GO analysis were used to classify the functions of the annotated DEGs under different temperatures treated for different number of days. In all comparisons, the most significantly enriched GO terms in biological process were "metabolic process", "cellular process", and "single-organism process". In cellular component, five GO terms including "cell", "cell part", "membrane", "membrane part", and "organelle" were highly enriched. "Binding" and "catalytic activity" which were in the molecular function were significantly enriched in all comparison's groups (Table 1). All DEGs were mapped to KEGG pathways in order to investigate the major pathways of them (Table 2). They were enriched in 132, 133, and 134 KEGG metabolic pathways in the $\mathrm{L} 2$ vs $\mathrm{H} 2$, L4 vs H4, and $\mathrm{L} 6$ vs $\mathrm{H} 6$ comparisons. In all the comparison group, "metabolic pathways", "biosynthesis of secondary metabolites", "plant-pathogen 


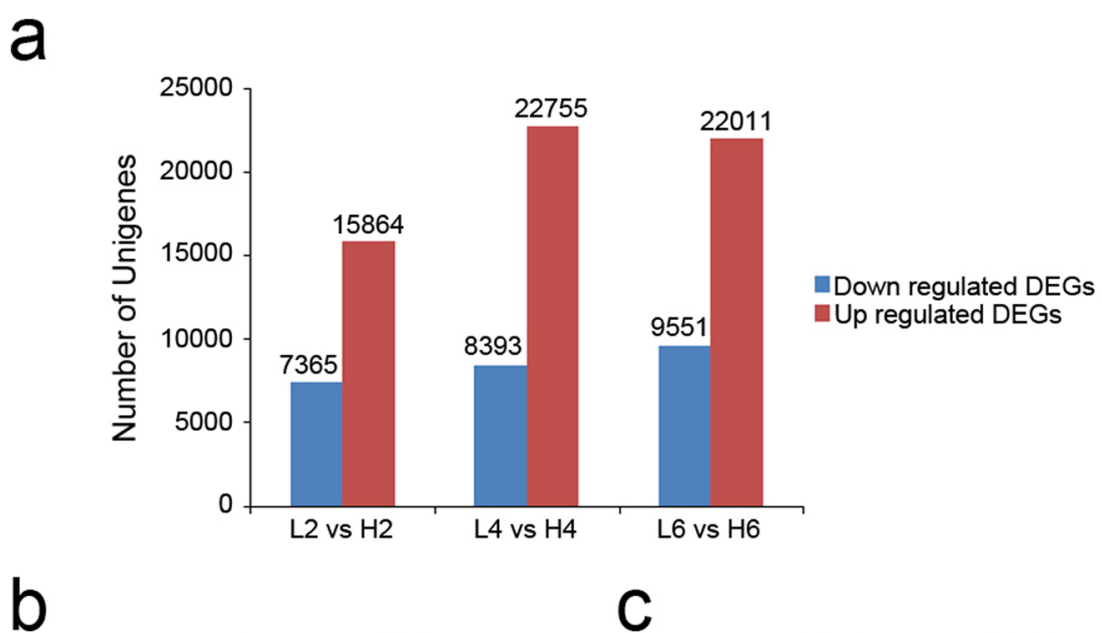

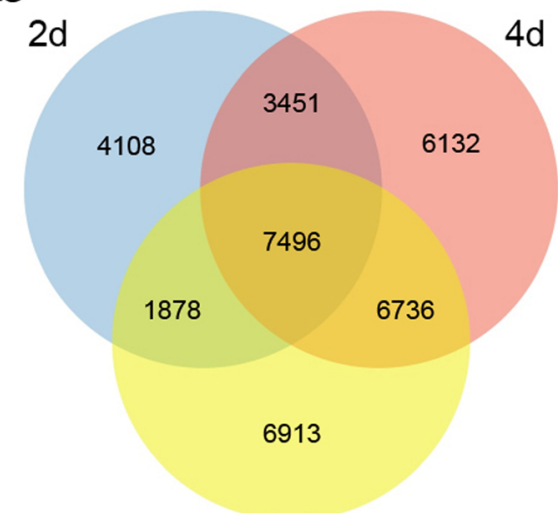

$6 d$

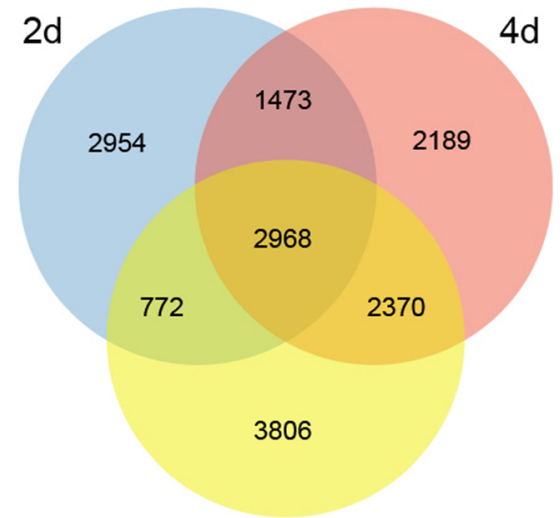

$6 d$

Fig. 3 Comparative analysis of DEGs under different temperatures treated by different days. a The number of up and down-regulated DEGs between the three comparisons ( $\mathrm{L} 2$ vs H2, L4 vs H4, and L6 vs H6). b Venn diagram of the number of up-regulated DEGs between the three comparisons. $\mathbf{c}$ Venn diagram of the number of down-regulated DEGs between the three comparisons. $L 2, L 4$ and $L 6$ respectively represents the samples from the plants under $19^{\circ} \mathrm{C}$ treatment for $2 \mathrm{~d}, 4 \mathrm{~d}$, and $6 \mathrm{~d} ; \mathrm{H} 2, \mathrm{H} 4$ and $\mathrm{H} 6$ respectively represents the samples from the plants under $23^{\circ} \mathrm{C}$ treatment for $2 \mathrm{~d}, 4 \mathrm{~d}$, and $6 \mathrm{~d}$

Table 1 The top ten most enriched GO terms of DEGs in all comparison groups

\begin{tabular}{|c|c|c|c|c|}
\hline \multirow{2}{*}{$\begin{array}{l}\text { GO term hierarchy } \\
1\end{array}$} & \multirow[t]{2}{*}{ GO term hierarchy 2} & \multicolumn{3}{|c|}{ Number of DEGs } \\
\hline & & L2 vs H2 & L4 vs H4 & L6 vs H6 \\
\hline \multirow[t]{3}{*}{ Biological process } & metabolic process & 1823 & 2328 & 2539 \\
\hline & cellular process & 1693 & 2190 & 2331 \\
\hline & single-organism process & 1117 & 1424 & 1556 \\
\hline \multirow[t]{5}{*}{ Cellular component } & cell & 1287 & 1626 & 1729 \\
\hline & cell part & 1271 & 1610 & 1710 \\
\hline & membrane & 1162 & 1395 & 1613 \\
\hline & membrane part & 886 & 1052 & 1229 \\
\hline & organelle & 866 & 1117 & 1187 \\
\hline \multirow[t]{2}{*}{ Molecular function } & binding & 1628 & 2135 & 2266 \\
\hline & catalytic activity & 1693 & 2136 & 2379 \\
\hline
\end{tabular}


Table 2 The top ten most enriched KEGG of DEGs in all comparison groups

\begin{tabular}{|c|c|c|c|c|c|c|c|}
\hline \multirow[t]{2}{*}{ Pathway } & \multirow[t]{2}{*}{ ID } & \multicolumn{2}{|l|}{$\mathrm{L} 2$ vs H2 } & \multicolumn{2}{|l|}{ L4 vs H4 } & \multicolumn{2}{|l|}{ L6 vs H6 } \\
\hline & & Percent (\%) & $P$-value & Percent (\%) & $P$-value & Percent (\%) & $P$-value \\
\hline Metabolic pathways & ko01100 & 22.94 & 3.69895E-07 & 23.64 & 2.02306E-16 & 23.78 & $9.35908 \mathrm{E}-20$ \\
\hline Biosynthesis of secondary metabolites & ko01110 & 12.43 & $1.83429 \mathrm{E}-07$ & 13.09 & 8.08594E-19 & 13.23 & 3.08193E-23 \\
\hline Plant-pathogen interaction & ko04626 & 5.42 & $2.90029 \mathrm{E}-05$ & 5.43 & 4.82036E-07 & 5.67 & 1.43759E-11 \\
\hline Starch and sucrose metabolism & ko00500 & 4.03 & $9.30016 \mathrm{E}-08$ & 3.69 & 3.81391E-05 & 3.77 & $8.46098 \mathrm{E}-07$ \\
\hline Plant hormone signal transduction & ko04075 & 3.96 & 0.01001351 & 4.27 & $6.50429 \mathrm{E}-07$ & 4.65 & $2.91758 \mathrm{E}-15$ \\
\hline Protein processing in endoplasmic reticulum & ko04141 & 3.04 & 0.03015519 & 2.82 & 0.2699489 & 2.63 & 0.827178 \\
\hline Carbon metabolism & ko01200 & 2.84 & 0.1309717 & 2.87 & 0.06273836 & 3.1 & 0.000191297 \\
\hline Endocytosis & ko04144 & 2.72 & 0.5626473 & 2.93 & 0.07581657 & 2.73 & 0.5247731 \\
\hline Ribosome & ko03010 & 2.76 & 0.006505746 & 2.94 & 4.06865E-06 & - & - \\
\hline Phenylpropanoid biosynthesis & ko00940 & 2.55 & $2.74168 \mathrm{E}-13$ & - & - & - & - \\
\hline Amino sugar and nucleotide sugar metabolism & ko00520 & 2.51 & 0.007218067 & 2.66 & $1.27859 \mathrm{E}-05$ & 2.46 & 0.003741373 \\
\hline Biosynthesis of amino acids & ko01230 & 2.45 & 0.8067985 & 2.66 & 0.272135 & 2.78 & 0.04693815 \\
\hline Spliceosome & ko03040 & - & - & 2.82 & 0.8755484 & 2.52 & 0.9998826 \\
\hline RNA transport & ko03013 & - & - & - & - & 2.56 & 0.997384 \\
\hline
\end{tabular}

Note: L2, L4 and L6 respectively represents the samples from the plants under $19{ }^{\circ} \mathrm{C}$ treatment for $2 \mathrm{~d}, 4 \mathrm{~d}$, and $6 \mathrm{~d}$; $\mathrm{H} 2, \mathrm{H} 4$ and $\mathrm{H} 6$ respectively represents the samples from the plants under $23^{\circ} \mathrm{C}$ treatment for $2 \mathrm{~d}, 4 \mathrm{~d}$, and $6 \mathrm{~d}$

interaction", "starch and sucrose metabolism", and "plant hormone signal transduction" were the top five pathways with the largest number of DEGs (Table 2).

\section{Identification of DEGs involved in the cell wall metabolism}

The expansion of petal cells depends on the cell wall loosening and cellulose biosynthesis, soluble carbohydrate allocation, ion and water transport, and cytoskeleton rearrangement [7]. Therefore, we screened the DEGs which may be involved in the cell wall metabolism under different temperatures. EXP and XTH, are two kinds of essential proteins involved in cell expansion, loosening and rearranging the cell wall fibers in growing tissues [8]. In cucumber, EXPs consist of four sub families: $\alpha$-EXP, $\beta$-EXP, EXP-like A and EXP-like B and were first discovered to loosen cell walls in $\mathrm{pH}$ dependent manner [27]. In our study, four $\alpha$-EXP and one $\beta$-EXP were significantly different expressed under $23^{\circ} \mathrm{C}$ and $19^{\circ} \mathrm{C}$ comparisons (Fig. 4, Table S8). Among them, four $\alpha$-EXP were up-regulated under relatively low temperature $\left(19^{\circ} \mathrm{C}\right)$ and one $\beta$-EXP was down-regulated under the treatment of $19^{\circ} \mathrm{C}$.

Eleven unigenes which encoded as XTH protein were significantly expressed, seven of them (CL256.Contig3, CL3252.Contig2, CL10571.Contig1, Unigene1021, Unigene1631, Unigene24045, and Unigene25288) were upregulated under the treatment of $19{ }^{\circ} \mathrm{C}$ and four (CL1547.Contig1, CL7741.Contig1, CL8777.Contig1, and
Unigene26777) of them were down-regulated under $19^{\circ} \mathrm{C}$ treatment (Fig. 4, Table S8).

The expression of unigenes involved in cell wall synthesis, modification or hydrolysis which probably participate in the process of flower opening were also assessed (Fig. 4, Table S8). There were three cellulose synthase (CES) genes, six xylosidase (XYL) genes, two pectin esterase (PE) genes, five polygalacturonase (PG) genes, and two pectate lyase (PL) genes with dramatic fold change in all three comparisons (Fig. 4, Table S8). The expression of all CESs and PLs genes increased when treated with relatively low temperature of $19^{\circ} \mathrm{C}$ for different number of days. Most of the XYLs were up-regulated under relatively low temperatures except Unigene17352. Three PGs' expression increased and two of them decreased when exposed to relatively low temperature. One PE gene was greatly up-regulated while one PE gene was down-regulated when the plants were exposed to $19^{\circ} \mathrm{C}$. Several genes associated with cell wall synthesis, modification or hydrolysis are up-regulated, suggesting that these genes may play vital roles in regulating petal cell expansion.

Aquaporins can facilitate the passage of water and/or small neutral solute fluxes across membranes and can be classified into four subclasses such as plasma membrane intrinsic proteins (PIPs), tonoplast intrinsic proteins (TIPs), nodulin-26-like intrinsic membrane proteins (NIPs) and small basic intrinsic proteins (SIPs) based on the sequence homology and cellular localization [28]. Three kinds of aquaporins including two PIPs, two TIPs 


\begin{tabular}{|c|c|c|c|c|c|c|c|}
\hline \multicolumn{3}{|c|}{ EXP } & \multicolumn{5}{|c|}{ CES } \\
\hline \multirow[t]{6}{*}{ L2 vs H2 } & L4 vs H4 & L6 vs $\mathrm{H}_{6}$ & & L2 vs H2 & L4 vs H4 & L6 vs $\mathrm{H} 6$ & \multirow{4}{*}{$\begin{array}{l}\text { CL4787.Contig2 } \\
\text { Unigene37789 } \\
\text { Unigene47737 }\end{array}$} \\
\hline & & & \multicolumn{4}{|l|}{ CL4845.Contig1 } & \\
\hline & & & \multicolumn{4}{|l|}{ CL8343_Contig1 } & \\
\hline & & & \multicolumn{4}{|l|}{ CL10934_Contig2 } & \\
\hline & & & \multicolumn{4}{|l|}{ CL12319_Contig2 } & \multirow{4}{*}{$\begin{array}{l}\text { Unigene21348 } \\
\text { CL1473_Contig2 }\end{array}$} \\
\hline & & & Unigene48446 & L2 vs H2 & L4 vs H4 & L6 vs H6 & \\
\hline \multirow{13}{*}{ L2 vs H2 } & XTH & & & & & & \\
\hline & L4 vs H4 & L6 vs $\mathrm{H} 6$ & & & & & \\
\hline & & & \multicolumn{4}{|l|}{ CL256_Contig3 } & \multirow{3}{*}{$\begin{array}{l}\text { Unigene9686 } \\
\text { Unigene17352 } \\
\text { CL10104_Contig1 }\end{array}$} \\
\hline & & & CL1547_Contig1 & & & & \\
\hline & & & \multicolumn{4}{|l|}{ CL3252_Contig2 } & \\
\hline & & & \multicolumn{4}{|l|}{ CL7741_Contig1 } & \multirow{4}{*}{$\begin{array}{l}\text { Unigene30444 } \\
\text { CL4510_Contig5 }\end{array}$} \\
\hline & & & CL87/7/Contig1 & L2 vs H2 & L4 vs H4 & L6 vs H6 & \\
\hline & & & \multicolumn{4}{|l|}{ CL10571_Contig1 } & \\
\hline & & & \multicolumn{4}{|l|}{ Unigene1021 } & \\
\hline & & & \multicolumn{4}{|l|}{ Unigene1631 } & \\
\hline & & & Unigene24045 & & & & \\
\hline & & & \multicolumn{4}{|l|}{ Unigene25288 } & Unigene9015 \\
\hline & & & \multicolumn{4}{|l|}{ Unigene 26777} & \multirow{3}{*}{$\begin{array}{l}\text { Unigene14618 } \\
\text { Unigene29828 } \\
\text { CL10006_Contig1 }\end{array}$} \\
\hline \multirow{10}{*}{ L2 vs H2 } & A guanorin & & & & & & \\
\hline & Aquaporin & 19 & & & \multicolumn{2}{|l|}{$\Omega_{0}$} & \\
\hline & & Lovs & \multirow{2}{*}{ CL1684_Contig1 } & & $P L$ & & \\
\hline & & & & L2 vs H2 & L4 vs $\mathrm{H} 4$ & L6 vs H6 & \multirow{3}{*}{$\begin{array}{l}\text { Unigene37135 } \\
\text { CL6542_Contig1 }\end{array}$} \\
\hline & & & \multicolumn{4}{|l|}{ CL7006_Contig1 } & \\
\hline & & & CL6991_Contig1 & & & & \\
\hline & & & Unigene10956 & & Rehydrin & & \\
\hline & & & CL8689_Contig2 & L2 vs H2 & L4 vs $\mathrm{H} 4$ & L6 vs H6 & \\
\hline & & & Unigene22675 & & & & Unigene46248 \\
\hline & & & Unigene40148 & & & & CL5294_Contig1 \\
\hline & & & & & & & Unigene53630 \\
\hline-14 & & 0 & & & & & Unigene2288 \\
\hline & & & & & & & CL8427_Contig1 \\
\hline $\begin{array}{l}\text { Fig. } 4 \text { Heat ma } \\
\text { represent the } u \\
\text { found in Table } \\
\text { polygalacturon } \\
\text { d; } \mathrm{H} 2, \mathrm{H} 4 \text { and }\end{array}$ & & & ples from the plants $\mathrm{u}$ & parisons (L & $\mathrm{S} \mathrm{H} 2, \mathrm{~L} 4$ vs $\mathrm{H}$ & nd $\mathrm{L} 6$ vs He & $\begin{array}{l}\text { ed rectangles } \\
\text { gene list can be } \\
\text {; PG: } \\
\text { for } 2 \mathrm{~d}, 4 \mathrm{~d} \text {, and } 6\end{array}$ \\
\hline
\end{tabular}

and three NIPs, were found in our transcriptomes and most of them were significantly up-regulated under relatively low temperature (Fig. 4, Table S8). However, only the expression level of two NIPs (Unigene22675, and Unigene40148) decreased under relatively low temperature. Dehydrins are hydrophilic, thermostable stress proteins and some of them were identified as osmotic stress-responsive protein which could facilitate the inflow and outflow of water [29]. In our research, all the dehydrin genes were down-regulated when exposed to relatively low temperature (Fig. 4, Table S8).
Identification of DEGs involved in the phytohormone signal transduction pathway

There are some reports on phytohormones that can affect the floral opening [19], and therefore, we explored the DEGs involved in eight phytohormone signal transduction pathways. The levels of endogenous AUX were much higher in the basal petals and application of exogenous indole-3-butyric acid (IBA) can enlarge the cell length in chrysanthemum [5]. Many unigenes involved in AUX signal transduction pathway were increasingly expressed with the flower opening of rose, suggesting 
that AUX signaling participated in petal development [30]. In this study, nineteen unigenes were significantly differently expressed under different temperatures in AUX signal transduction pathway which included the auxin transporter protein (AUX1), auxin-induced protein/auxinresponsive protein (AUX/IAA), auxin response factor (ARF), auxin responsive GH3 family protein (GH3) and small auxin-up RNA family protein (SAUR) (Fig. 5). The majority of them had lower expression under relatively low temperature, especially three ARF members (CL661.Contig9, CL274.Contig1, and CL12908.Contig6), which decreased sharply as the treated days lengthened. Only one AUX1, two AUX/IAA, one ARF, and one GH3 were up-regulated when treated with relatively low temperature (Fig. 5, Table S9).

The phytohormone JA and its derivative methyl jasmonate (MeJA) can affect the flower opening of $E$. grandiflorum [31], rice [32], A. thaliana [20]. The gene BIGPETALP (BPEP), which can be regulated by JA, may also play a role in JA-mediated petal growth [6]. In JA signal transduction pathway, most of genes such as Coronatine-insensitive protein 1 (COI1), jasmonate ZIM domain protein $(J A Z)$, and $M Y C 2$ were down-regulated in this study. Only two JAZ (Unigene43759 and CL8754.Contig3) genes and four MYC2 (CL37.Contig1, CL6240.Contig1, CL3114.Contig2, and CL2658.Contig2) genes were up-regulated under $19^{\circ} \mathrm{C}$ treatment (Fig. 5, Table S9), suggesting that these unigenes may function as positive regulators of flower opening in O. fragrans (Fig. 5, Table S9). Only six TGACG-sequence-specific DNA-binding protein (TGA) genes in salicylic acid (SA) signal transduction pathway were expressed differently under different temperatures and two of them (CL2000.Contig1 and CL225.Contig1) were increased after $19^{\circ} \mathrm{C}$ treatment (Fig. 5, Table S9).

Nineteen unigenes in the gibberellin acid (GA) signaling pathway were also differentially expressed under different temperatures (Fig. 5). Five of six GID1 were down-regulated under relatively low temperature, with the exception of Unigene22649, which was nearly increased eight thousand times after $2 \mathrm{~d}$ treatment of $19^{\circ} \mathrm{C}$. Three DELLA and five PIF3 genes were decreased while two DELLA and three PIF3 genes were increased after relatively low temperature treatment (Fig. 5, Table S9). Only four of twenty unigenes including two PYR/ PYL and two ABF (ABA responsive element binding

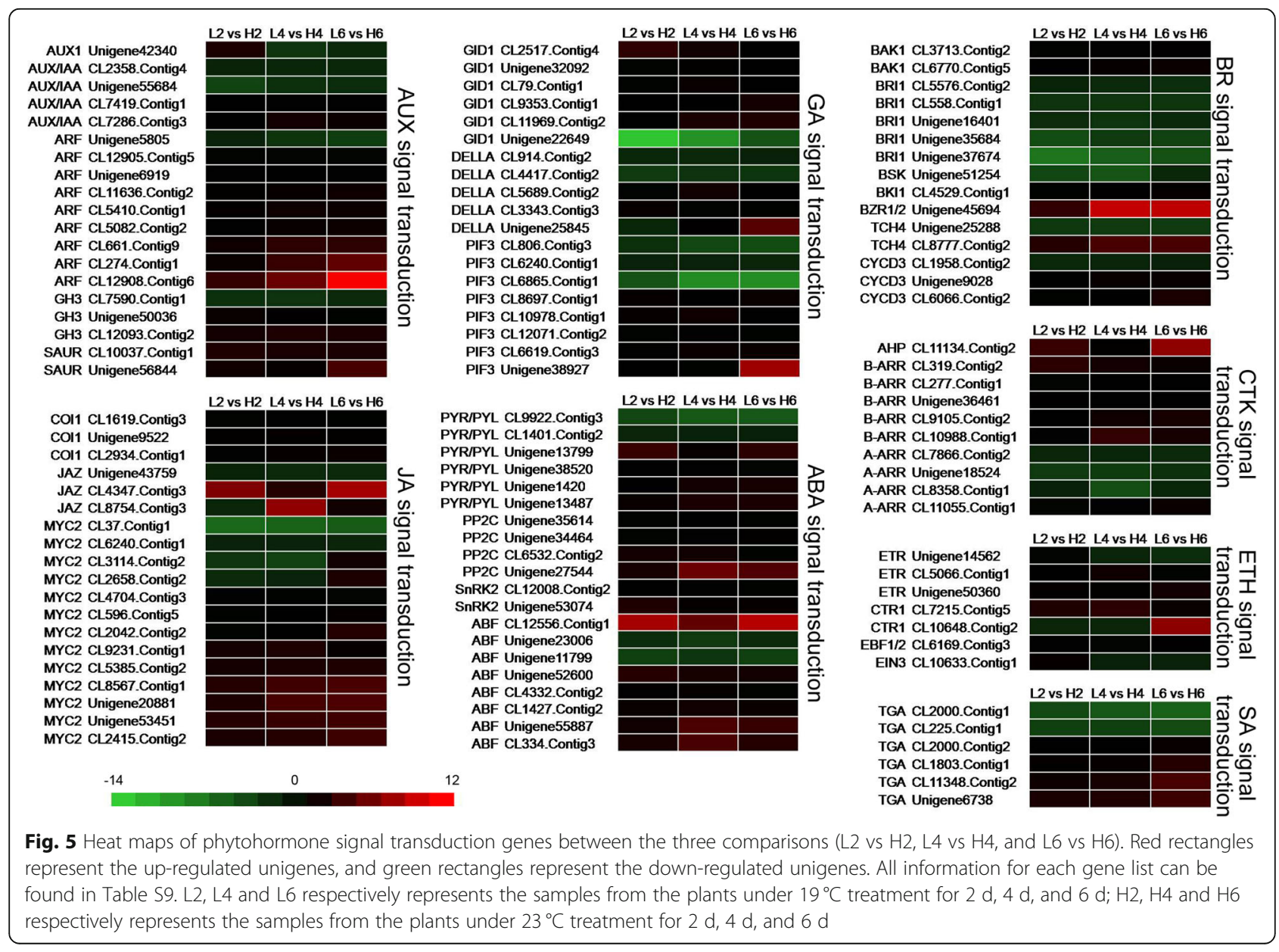


factor) genes in ABA signal transduction pathway were up-regulated after $19^{\circ} \mathrm{C}$ treatment. However, the expression of one ABF (CL12556.Contig1) was decreased by two hundred times under the relatively low temperature at $19^{\circ} \mathrm{C}$ compared with that under $23^{\circ} \mathrm{C}$ (Fig. 5, Table S9).

Most of the unigenes in cytokinin (CTK) signal transduction pathway were down-regulated under relatively low temperature including all Arabidopsis histidine phosphotransfer Protein (AHP) genes, and all B-ARR members, especially the AHP (CL11134.Contig2), which was decreased severely after $6 \mathrm{~d}$ treatment of $19^{\circ} \mathrm{C}$ (Fig. 5). However, most of the A-ARR members (CL7866.Contig2, Unigene18524, and CL8358.Contig1) were up-regulated when treated by relatively low temperature (Fig. 5, Table S9).

The effect of ETH on flower opening may be dosedependent, species-dependent or cultivar-dependent [19]. In ETH signal transduction pathway, the expression pattern of some ethylene receptor (ETR, Unigene14562), serine/threonine-protein kinase (CTR1, CL10648.Contig2), and ethylene insensitive 3 protein (EIN3, CL10633.Contig1) genes, were more complicated. For example, the expression level of Unigene14562 and CL10633.Contig1 were decreased when treated by $2 \mathrm{~d}$ of relatively low temperature, while they were increased when the treatment time was lengthened. Most of the genes in this pathway were down-regulated after the relatively low temperature' treatment (Fig. 5, Table S9).

Cell elongation is also regulated by $\mathrm{BR}$, which is a plant-specific steroid hormone and longitudinal cell expansion of BR mutants is greatly reduced in A. thaliana $[33,34]$. Eight of fifteen unigenes in the BR signal transduction pathways were increased under $19^{\circ} \mathrm{C}$ treatment, including all brassinosteroid receptor (BRI1), one BRI1 Associated receptor Kinase 1 (BSK), one TCH4, and one cyclin-D3 (CYCD3) (Fig. 5, Table S9). The expression level of BRI1, especially Unigene37674 and Unigene35684 were dramatically increased when treated with $19^{\circ} \mathrm{C}$. However, seven of fifteen unigenes in this signal pathway were decreased under $19^{\circ} \mathrm{C}$ treatment. The expression level of BZR1/2 was eight-fold under $19^{\circ} \mathrm{C}$ compared to $23^{\circ} \mathrm{C}$, and the expression level were continuously increased as the treatment time prolonged, suggesting the same role of BZR1/2 in petal development of O. fragrans and G. hybrida. Similarly, the expression level of TCH4 (CL8777.Contig2) was enhanced by relatively low temperature.

\section{Identification of DEGs of transcription factors (TFs)}

Many TFs changed dramatically under different temperatures, so we improved the screening criteria. The TFs with a $\mid \log _{2}$ Ratio $\mid \geq 2$ in any comparison were analyzed further. 79 TFs which were divided into eight gene family (Fig. 6, Table S10) were selected for further analysis because they may be involved in petal cell expansion [5, 30]. In the MYB TF family, only five out of twenty genes (Unigene36971, CL242.Contig2, CL10695.Contig2, Unigene11061, and Unigene30676) were up-expressed under relatively low temperature. Overexpression of the MYB62 gene results in a GA-deficient phenotype and can be partially alleviated with the application of exogenous GA [35]. In our study, we did not find the homologous unigenes to MYB62 in O. fragrans, but some unigenes homologous to other gene family members, such as MYB4 (CL9450.Contig1) and MYB86 (Unigene19015), were expressed differently under different temperatures (Fig. 6, Table S10), indicating they may be involved in the regulation of relatively low temperature on flowering opening in O. fragrans.

In the bHLH TF family, three genes (Unigene30441, CL796.Contig2, and CL6865.Contig1) were up expressed after treated by $19^{\circ} \mathrm{C}$. Among them, especially the bHLH79, the expression level of bHLH79 isoform X2 (CL6865.Contig1) increased five hundred times when treated by $6 \mathrm{~d}$ of relatively low temperature. However, the expression level of bHLH 117 decreased sharply when exposed to $19^{\circ} \mathrm{C}$. None of the TF genes was up-regulated under relatively low temperature in the AP2 and NAC TF family. Plant-specific NAC family genes can regulate plant development, cell division, senescence, and responses to abiotic stress [36]. In recent research, the N-terminal binding domain of RhNAC2 could be bound to the promoter of RhEXPA4 thus regulating of dehydration tolerance during the expansion of rose petals [37]. The expression of all the members of NAC family decreased under the relatively low temperature (Fig. 6, Table S10), indicating that the suppression of NAC genes may facilitate the expression of EXPs to promote the elongation of flower petals in $O$. fragrans.

In the TCP TF family, two genes (CL9936.Contig2, and Unigene10400) were up-expressed under relatively low temperature treatment. miRNAs, such as miRNA319a, have also been found to be involved in petal elongation by regulating TCP4 which regulates AUX effects [38]. Therefore, TCP4-like gene (CL9936.Contig2, and Unigene10400) increased with relatively low temperature treatment, implying they may regulate the petal development in O. fragrans (Fig. 6, Table S10).

Four out of nine genes were up-regulated in the MADS TF family, especially the AGAMOUS gene, which is the $\mathrm{C}$-function genes, and its expression increased significantly with the $19^{\circ} \mathrm{C}$ treatment. In the WRKY TF family, only two genes (CL11608.Contig1, and Unigene860) were up-regulated under relatively low temperature. In Zinc finger TF family, four out of eleven genes' expression increased when exposed to $19^{\circ} \mathrm{C}$. Generally speaking, only a small proportion of TFs were upregulated under relatively low temperature. 


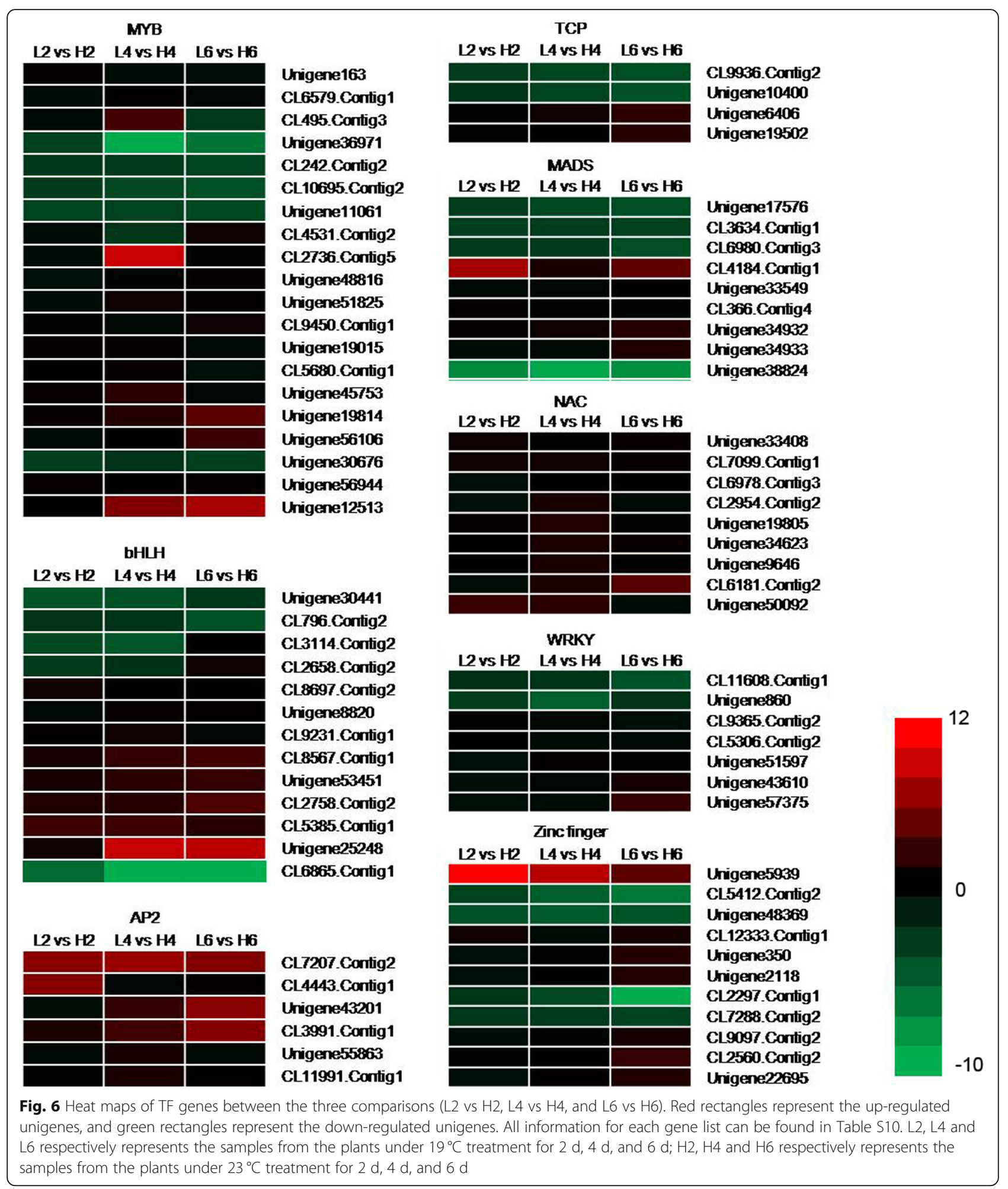

qRT-PCR analysis

Ten differentially expressed unigenes comprising four genes involving in cell wall metabolism, four genes related to phytohormone signal transduction and two genes of TF were analyzed by qRT-PCR in order to confirm the results of RNA-Seq. All ten genes tested by qRT-PCR confirmed significant differential expression $(P<0.05)$ between $19{ }^{\circ} \mathrm{C}$ and $23^{\circ} \mathrm{C}$ treatments (Fig. 7). Although fold changes of the ten genes between $19^{\circ} \mathrm{C}$ and $23^{\circ} \mathrm{C}$ treatments were not always similar in RNA- 


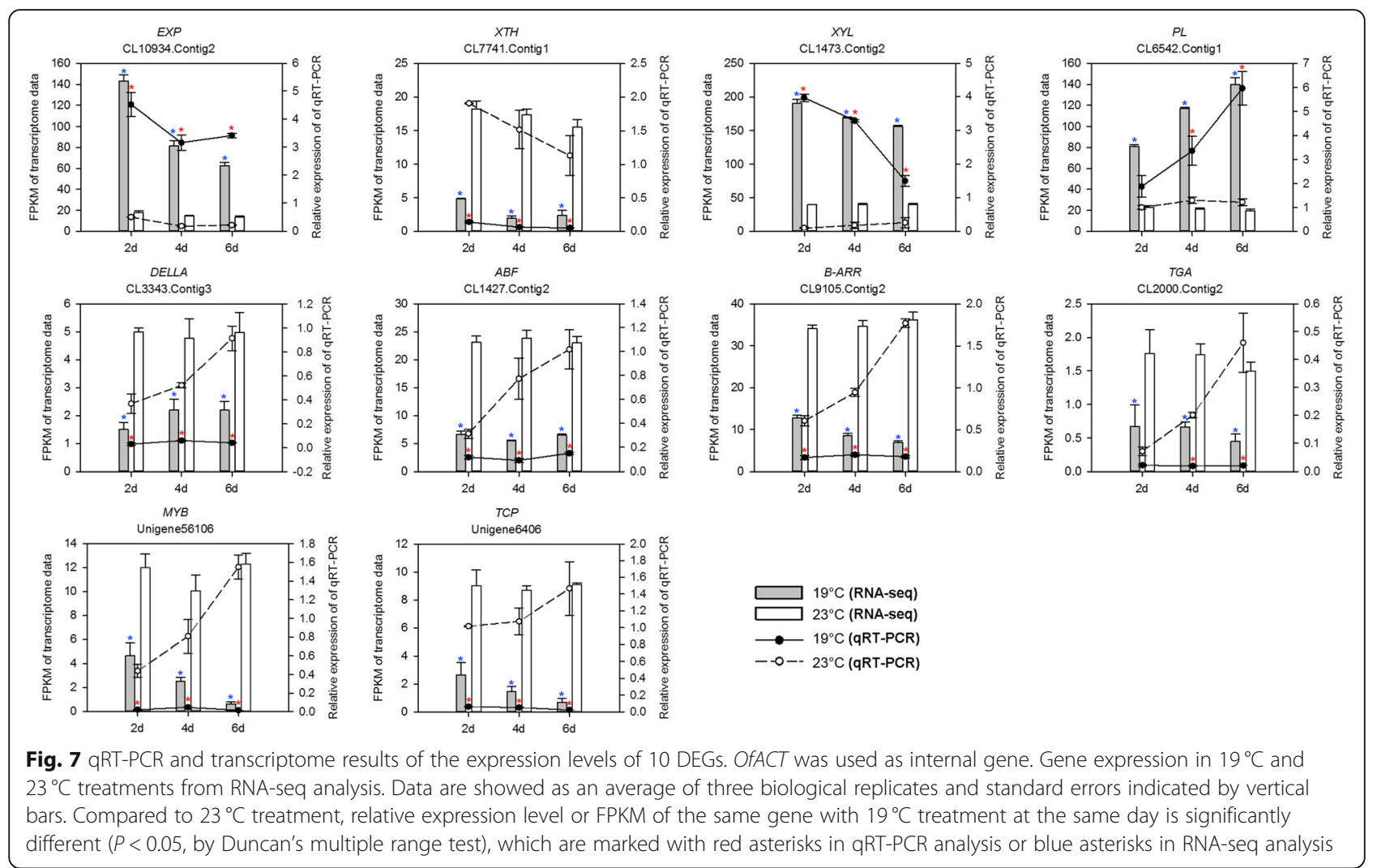

Seq and qRT-PCR results, the overall trend was consistent. Generally, the expression levels of CL10934.Contig2 (EXP), CL1473.Contig2 (XYL) and CL6542.Contig1 (PL) were significantly lower in $23^{\circ} \mathrm{C}$ treatment than those in $19^{\circ} \mathrm{C}$ treatment with qRT-PCR analysis, while the expression levels of other seven genes were higher in $23^{\circ} \mathrm{C}$ treatment. Similarly, CL10934.Contig2, CL1473.Contig2 and CL6542.Contig1 were down-regulated, while other seven genes were up-regulated in $23^{\circ} \mathrm{C}$ treatment, which verified the accuracy of RNA-Seq results.

\section{Discussion}

The flowering time of once-flowering cultivars of $O$. fragrans is greatly affected by the relatively low temperature and they need a certain amount of low temperature accumulation at autumn before blooming [3]. The sensitivity difference to the requirement of relatively low temperature among different cultivars leads to the variation of flowering time [3]. In this study, $19^{\circ} \mathrm{C}$ treatment in experimental condition for $6 \mathrm{~d}$ is enough for flower opening in the cultivar 'Yanhong Gui'. The early flowering cultivar such as 'Zao Yingui' would blossom when the daily minimum temperature lower than $21^{\circ} \mathrm{C}$ last for after 5-8 days, while the late blossoming cultivar, such as 'Wan Yingui', needs 5-8 days of the daily minimum temperature lower than $19^{\circ} \mathrm{C}$ [39].
Flower opening is accompaning with cell division and cell expansion in petal cells [7]. are up-regulated during floral open process in sandersonia [40], Mirabilis jalapa [41], petunia [9], carnation [42], and wintersweet [10]. Similarly, during flower opening of $O$. fragrans under relatively low temperature, four $\alpha$-EXP were upregulated in this study (Fig. 4, Table S8). It was also reported that $\beta$-EXP is more efficient in disrupting cell wall polymers than $\alpha$-EXP [41], however, one $\beta$-EXP was down-regulated under $19^{\circ} \mathrm{C}$ treatment. The result is coincidence with the results in rose [30], suggesting that $\beta$ EXP may be involved in petal development in a special manner different from $\alpha$-EXP. As to XTH, four rose $X T H$ genes (RbXTH3, RbXTH5, RbXTH6, and $R b X T H 12)$ were up-regulated during flower opening and associated with petal movement [43]. Rice OsXTH12 gene can cause rice glume-unclosing after anthesis under high temperature conditions [44]. In our experiment, we cannot found the homologous genes of XTH3, XTH5, $X T H 6$ and $X T H 12$, but other family members were discovered. Most of XTH genes were up-regulated under relatively low temperature, indicating that they may be involved in the flower opening process of $O$. fragrans and different family members may have different functions.

These cell wall metabolism processes were also influenced by multiple kinds of phytohormones via 
transcription factors [19]. The expression patterns of phytohormone pathway genes are different under different temperatures treated by different days in our research. In A. thaliana, both the AUX and JA signal transduction function synergistically during petal development [45]. And ARF8 can interacts with BIGPETALp (BPEp) to regulate cell expansion in petals [6] and can trigger the expression of MYB21 and MYB24 to induce the production of JA to promote petal growth [45]. However, most DEGs involved in AUX and JA signal pathways were down-regulated (Fig. 5, Table S9), suggesting that the regulation of relatively low temperature on flower opening in $O$. fragrans doesn't depend on AUX and JA signaling.

GA can regulate seed germination, stem elongation, petal growth and flowering [46], and ABA has an antagonistically role such as in floral transition and fruit development [47]. The same circumstance occurred in the G. hybrida petal growth [22]. Compared to unigenes involved in ABA signal transduction pathway, there were much more unigenes in GA signal transduction pathway which increased sharply under the treatment of $19^{\circ} \mathrm{C}$. This result was consistent with the result in rose [30]. However, whether this kind of antagonistic role exists in petal growth of $O$. fragrans still needs further demonstration.

ETH can promote flower opening in carnation, Phalaenopsis orchids, and petunia, but it inhibits flower opening in several rose cultivars [19]. ETH can induce the expression of DELLA genes via EIN3-3 when it functions as inhibitors during flower opening in rose [22]. In our study, EIN3 was down-regulated under relatively low temperature (Fig. 5, Table S9), suggesting that ETH may function negatively on flower opening in response to relatively low temperature in $O$. fragrans.

In G. hybrida, BL shows more efficient effect on lengthening the cells in the middle and basal regions of petals than GA [21]. But the expression level of BES1/ BZR1 was decreased after only $0.5 \mathrm{~h}$ BR treatment, indicating its vital role in petal cell expansion [21]. In our study, expression level of the homologous unigene of BZR1 (Unigene45694), decreased dramatically when exposed to relatively low temperature (Fig. 5, Table S9), suggesting the same role in petal development of $O$. fragrans as that in G. hybrida.

\section{Conclusion}

The comprehensive transcriptomic dataset relating to flower opening of $O$. fragrans under relatively low temperature $\left(19^{\circ} \mathrm{C}\right)$ treatment was performed using RNA-seq technology, and a group of DEGs, which may regulate petal growth through pairwise comparison analysis of DEGs between different temperature treatments, had been identified, including unigenes involved in cell wall metabolism, phytohormone signal transduction pathway, and TFs. Overall, the resources generated in this study would lay foundation on fully revealing the molecular mechanism of relatively-low temperatureregulated flower opening of $O$. fragrans and provide theoretical reference for the prediction and regulation of flowering time and genetic modification in O. fragrans. Our future research will be focused on understanding the biological function of these candidate important DEGs and revealing how these DEGs response to relatively low temperature in O. fragrans.

\section{Methods}

\section{Plant materials and treatments}

Plants of $O$. fragrans 'Yanhong Gui' were potted and grown in the resource nursery of Zhejiang Agriculture and Forestry University. All plant materials were owned by Zhejiang Agriculture and Forestry University. The development of flower opening process are classified into S1-S6 as described in this paper [3]. When the floral development stage was at S1, potted plants were moved into the growth cabinet with the temperature of $19^{\circ} \mathrm{C}$ (relatively low temperature) or $23^{\circ} \mathrm{C}$ (relative high temperature), respectively. The relative humidity was about $50-60 \%$ and the photoperiod was $12 \mathrm{~h} \mathrm{light} / 12 \mathrm{~h}$ dark regime with an illumination of $80 \mu \mathrm{mol} \cdot \mathrm{m}^{-2} \cdot \mathrm{s}^{-1}$. Petals were harvested every 2 days after treatment until the developmental stage of plants under $19^{\circ} \mathrm{C}$ condition reached S2. The samples were named as $\mathrm{H} 2, \mathrm{H} 4, \mathrm{H} 6$ (treated for $2 \mathrm{~d}, 4 \mathrm{~d}$, and $6 \mathrm{~d}$ under $23^{\circ} \mathrm{C}$ treatment, respectively) and L2, L4, L6 (treated by $2 \mathrm{~d}, 4 \mathrm{~d}$, and $6 \mathrm{~d}$ under $19^{\circ} \mathrm{C}$ treatment, respectively). All samples were collected at 10:00 am in order to avoid the influence of diurnal rhythm. Three biological replicate samples were collected to generate 18 samples in total. In addition, petals at different developmental stages (S1-S6) were respectively sampled for SEM and TEM analysis.

\section{SEM and TEM analysis}

According to previous studies [25, 48], SEM and TEM were carried out and were observed and photographed in Hitachi Model TM-1000 SEM and Model H-7650 respectively in Zhejiang University. Cell size of adaxial and abaxial petal epidermal cells, and vacuole size were manually measured using Image J software (http://rsb. info.nih.gov/ij/, NIH, MD, USA).

RNA extraction, CDNA library preparation and sequencing RNA isolation and RNA purification of 18 samples were carried out as described previously [49]. The cDNA library preparation and sequencing of 18 samples of $O$. fragrans were respectively performed as described previously [50] for RNA-seq analysis. What's more, a RNA pool mixed from 18 RNA samples was used to construct 
a cDNA library for reference transcriptome sequencing. The Illumina sequencing was performed at the Beijing Genomics Institute (BGI) (Shenzhen, China) (http:// www.genomics.cn/index.php) according to the manufacturer's instructions (Illumina Inc., San Diego, CA, USA). A total of 18 sets of raw reads were obtained for RNAseq analysis, corresponding to $\mathrm{H} 2-1, \mathrm{H} 2-2, \mathrm{H} 2-3, \mathrm{H} 4-$ 1, H4-2, H4-3, H6-1, H6-2, H6-3, L2-1, L2-2, L2-3, L4-1, L4-2, L4-3, L6-1, L6-2, and L6-3, while raw reads were obtained for reference transcriptome corresponding to ALL.

\section{Transcriptome data processing and analysis}

Raw reads were cleaned by removing the sequence of adaptor, high content of unknown bases and low-quality reads before downstream analysis to decrease data noise. As $O$. fragrans did not have an appropriate reference genome sequence, the Trinity method [51] was used to de novo assemble all clean reads and the Tgicl method [52] was used to cluster the transcripts to obtain nonredundant unigenes. The assembled unigene sequences were aligned by Blastn [53] to nucleotide database NT, aligned by Blastx [53] or Diamond [54] to protein databases NR, Swiss-Prot, KEGG, and KOG, and aligned by InterProScan5 [55] to protein database InterPro to get the annotations. With the NR annotation, GO annotations of unigenes were obtained using Blast2GO software [56]. FPKM (reads mapped per 1000 bp per million sequenced reads) method was used in calculated expression level [57]. Comparisons of FPKM between samples treated under different temperatures for the same number of days (L2 vs H2, L4 vs H4, and L6 vs H6 respectively) were performed for each unigene. To identify the DEGs in the two samples, the Audic and Claverie method was employed [58]. Unigene with a $P$ value $\leq 0.01$ and a $\mid \log _{2}$ Ratio $\mid \geq 1$ were considered significant DEGs. All DEGs were mapped to each term of the GO and KEGG databases. For the analysis of transcription factors, $\mid \log _{2}$ Ratio $\mid \geq 2$ were marked to be significantly different between the two samples.

\section{Quantitative real-time PCR (qRT-PCR) analysis}

The extraction of total RNA, first-strand cDNA synthesis, and qRT-PCR were performed as previously described [49]. Ten DEGs were randomly chosen and the primer sequences were listed in Table S11. Gene relative expression levels of those ten genes were normalized relatively to the expression level of an internal control OfACT [59] using the $2^{-\triangle \mathrm{Ct}}$ method.

\section{Statistical analysis}

The statistical analysis of the size of adaxial petal epidermal cells, abaxial petal epidermal cells, and vacuole, as well as relative expression level and FPKM analysis was performed by one-way analysis of variance (ANOVA) using SPSS software version 18.0 (SPSS Inc., Chicago, IL, USA). Duncan's multiple range test was employed and differences of $P<0.05$ were considered significant.

\section{Supplementary information}

Supplementary information accompanies this paper at https://doi.org/10. 1186/s12870-020-02549-3.

Additional file 1: Figure S1. A species-based distribution of BLASTX matches for unigenes from the reference transcriptome of Osmanthus fragrans. We used all the plant proteins in the NCBI NR database to perform the homology search and for each sequence we selected the closest match for analysis.

Additional file 2: Figure S2. GO classification of unigenes from the reference transcriptome of Osmanthus fragrans. Results are summarized under three main $\mathrm{GO}$ categories: biological process, cellular component and molecular function. The right $x$-axis indicates the number of genes in the same category.

Additional file 3: Figure S3. KOG classification of Osmanthus fragrans unigenes from the reference transcriptome. From a total of 96,920 de novo assembled unigenes, 43,496 transcripts with significant homologies in the KOG database were classified into 25 KOG categories.

Additional file 4: Table S1. The size of adaxial petal epidermal cells, abaxial petal epidermal cells, and vacuole of adaxial petal epidermal cells in sweet osmanthus flowers at different developmental stages

Additional file 5: Table S2. Summary of Illumina transcriptomic sequencing.

Additional file 6: Table S3. Summary of assembly quality of transcripts and unigenes.

Additional file 7: Table S4. Annotation results of the assembled unigenes from the reference transcriptome against public databases.

Additional file 8: Table S5. KEGG pathway annotation of Osmanthus fragrans unigenes obtained from the reference transcriptome. There were 40,764 unigenes mapped into 134 KEGG pathways.

Additional file 9: Table S6. Number of total clean reads of the 18 samples mapped to reference sequences.

Additional file 10: Table S7.Pearson correlation coefficients of six samples each with three biological replicates.

Additional file 11: Table S8. DEGs involved in the cell wall metabolism under different temperatures treated by different days in Osmanthus fragrans.

Additional file 12: Table S9. DEGs involved in the phytohormone signal transduction pathway under different temperatures treated by different days in Osmanthus fragrans.

Additional file 13: Table S10. DEGs involved in TFs under different temperatures treated by different days in Osmanthus fragrans.

Additional file 14: Table S11. Primer sequences and amplicon characteristics.

\section{Abbreviations}

ABA: Abscisic acid; ARF: Auxin response factor; AHP: Arabidopsis histidine phosphotransfer protein; AUX: Auxin; AUX/IAA: Auxin-induced protein/auxinresponsive protein; AUX1: Auxin transporter protein; BPEp: BIGPETALp; BR: Brassinosteroid; CES: Cellulose synthase; COI1: Coronatine-insensitive protein 1; CYCD3: Cyclin-D3; CTK: Cytokinin; CTR1: Serine/threonine-protein kinase; DEGs: Differentially expressed genes; EIN3: Ethylene insentitive 3 protein; ETH: Ethylene; ETR: Ethylene receptor; EXP: Expansin; FPKM: Reads mapped per 1000 bp per million sequenced reads; GA: Gibberellin; GH3: Auxin responsive GH3 family protein; IAA: Indole-3-acetic acid; IBA: Indole-3-butyric acid; JA: Jasmonic acid; JAZ: Jasmonate ZIM domain protein; KEGG: Kyoto Encyclopedia of Genes and Genomes; KOG: Eukaryotic Orthologous Groups of proteins; MeJA: Methyl jasmonate; NIP: Nodulin-26like intrinsic membrane protein; NR: Non-Redundant Protein Sequence 
Database; NT: Nucleotide Sequence Database; GA: Gibberellin acid; GO: Gene Ontology; PE: Pectin esterase; PG: Polygalacturonase; PIP: Plasma membrane intrinsic protein; PL: Pectate lyase; qRT-PCR: Quantitative real-time PCR; SA: Salicylic acid; SAUR: Small auxin-up RNA family protein; SEM: Scanning electron microscopy; SIP: Small basic intrinsic protein; TGA: TGACG-sequencespecific DNA-binding protein; TEM: Transmission electron microscopy; TIP: Tonoplast intrinsic protein; XTH: Xyloglucan endotransglycosylase/ hydrolase; XYL: Xylosidase

\section{Acknowledgements}

Not applicable.

\section{Consent to publish}

Not applicable.

\section{Authors' contributions}

$\mathrm{HZ}$ designed the research. JF, CZ and $\mathrm{HZ}$ drafted the manuscript. JF and CZ performed the transcriptome data processing and analysis. $Y L$ performed qRT-PCR detection. TP extracted RNA and constructed CDNA Library. BD, XG and YZ performed SEM and TEM analysis. All authors have read and approved the manuscript.

\section{Funding}

This research was supported by Zhejiang Provincial Natural Science Foundation of China (Grant Nos. LY19C160006, LY19C160002, and LQ16C160003), Zhejiang Provincial Major Program of New Cultivar Breeding (Grant No. 2016C02056-12), and China Scholarship Council (201808330145 and 201808330146). The funding organizations provided the financial support to the research projects, but were not involved in the design of the study, data collection, analysis of the data, or the writing of the manuscript.

\section{Availability of data and materials}

The RNA-Seq datasets used in the current study are available on the NCB Short Read Archive Project - PRJNA642321 (http://www.ncbi.nlm.nih.gov/bioproject/PRJNA642321). All RNA-seq reads generated by this study are publicly available at the NCBI SRA under accession numbers SRR12109992-

SRR12110009

\section{Ethics approval and consent to participate}

Not applicable.

\section{Competing interests}

The authors declare that they have no competing interests.

Received: 5 April 2020 Accepted: 12 July 2020

Published online: 16 July 2020

\section{References}

1. Yang K. Chinese Osmanthus. Beijing: China Forestry Publication House; 2011.

2. Xiang Q, Liu Y. An illustrated monograph of the sweet osmanthus cultivars in China. Hangzhou: Zhejiang Science and Technology Press; 2008.

3. Wang Y, Zhang C, Fu J, Zhao H. Progresses on flower bud differentiation and flower opening in Osmanthus fragrans. J Zhejiang A \& F Univ. 2016; 33(2):340-7.

4. Dong L, Wang X, Ding Y. Study on the Osmanthus fragrans blooming season phenology. J Nanjing Forestry Univ (Nat Sci Ed). 2014;38(S1):51-6.

5. Wang J, Wang H, Ding L, Song A, Shen F, Jiang J, Chen S, Chen F. Transcriptomic and hormone analyses reveal mechanisms underlying peta elongation in Chrysanthemum morifolium 'Jinba'. Plant Mol Biol. 2017;93(6): 593-606.

6. Varaud E, Brioudes F, Szecsi J, Leroux J, Brown S, Perrot-Rechenmann C, Bendahmane M. AUXIN RESPONSE FACTOR8 regulates Arabidopsis petal growth by interacting with the bHLH transcription FACTOR BIGPETALp. Plant Cell. 2011:23(3):973-83.
7. Christiaens A, De KE, Pauwels E, De RJ, Gobin B, Van Labeke MC. Suboptimal light conditions influence source-sink metabolism during flowering. Front Plant Sci. 2016;7:249

8. Cosgrove DJ. Wall structure and wall loosening. A look backwards and forwards. Plant Physiol. 2001;125(1):131-4.

9. Zenoni S, Reale L, Tornielli GB, Lanfaloni L, Porceddu A, Ferrarini A, Moretti C, Zamboni A, Speghini A, Ferranti F. Downregulation of the Petunia hybrida alpha-expansin gene PhEXP1 reduces the amount of crystalline cellulose in cell walls and leads to phenotypic changes in petal limbs. Plant Cell. 2004;16(2):295-308.

10. Ma J, Li Z, Wang B, Sui S, Li M. Cloning of an expansin gene from Chimonanthus praecox flowers and its expression in flowers treated with ethephon or 1-methylcyclopropene. Hortscience. 2012;47(10):1472-7.

11. Panavas T, Reid PD, Rubinstein B. Programmed cell death of daylily petals: activities of wall-based enzymes and effects of heat shock. Plant Physiol Biochem. 1998;36(5):379-88.

12. Tarpley L, Sassenrath GF. Carbohydrate profiles during cotton floral bud (square) development. J Agron Crop Sci. 2006;192(5):363-72.

13. Ichimura KK, Koketsu M, Shimamura M, Ito A. Identification of pinitol as a main sugar constituent and changes in its content during flower bud development in carnation (Dianthus caryophyllus L.). J Plant Physiol. 1998; 152(5):363-7.

14. Yamada K, Ichimura K. Determination of subcellular concentrations of soluble carbohydrates in rose petals during opening by nonaqueous fractionation method combined with infiltration-centrifugation method. Planta. 2009;230(6):1115-27.

15. Ichimura K, Kohata K, Yamaguchi Y, Douzono M, Ikeda H, Koketsu M. Identification of L-inositol and scyllitol and their distribution in various organs in chrysanthemum. Biosci Biotechnol Biochem. 2000;64(4):865-8.

16. Norikoshi R, Imanishi H, Ichimura K. Changes in cell number, osmotic potential and concentrations of carbohydrates and inorganic ions in Tweedia caerulea during flower opening. J Jpn Soc Hortic Sci. 2013;82(1):516.

17. Norikoshi R, Kohata K, Shimizu-Yumoto H, Goto R, Ichimura K. Identification of soluble carbohydrates and their subcellular concentrations in petals during flower opening in Eustoma grandiflorum. Horticult J. 2016;85(3):23847.

18. Krizek BA, Anderson JT. Control of flower size. J Exp Bot. 2013;64(6):1427-37.

19. van Doorn WG, Kamdee C. Flower opening and closure: an update. J Exp Bot. 2014:65(20):5749-57.

20. Brioudes F, Joly C, Szécsi J, Varaud E, Leroux J, Bellvert F, Bertrand C, Bendahmane M. Jasmonate controls late development stages of petal growth in Arabidopsis thaliana. Plant J. 2009;60(6):1070-80.

21. Huang G, Han M, Yao W, Wang Y. Transcriptome analysis reveals the regulation of brassinosteroids on petal growth in Gerbera hybrida. Peerj. 2017:5(5):e3382.

22. Li L, Zhang W, Zhang L, Li N, Peng J, Wang Y, Zhong C, Yang Y, Sun S, Liang S. Transcriptomic insights into antagonistic effects of gibberellin and abscisic acid on petal growth in Gerbera hybrida. Front Plant Sci. 2015;6:168.

23. Koning RE. The roles of plant hormones in the growth of the corolla of Gaillardia grandiflora (Asteraceae) ray flowers. Am J Bot. 1984;71(1):1-8.

24. Kenis JD, Silvente ST, Trippi VS. Nitrogen metabolism and senescenceassociated changes during growth of carnation flowers. Physiol Plantarum. 1985;65(4):455-9. 
25. Yamada K, Norikoshi R, Suzuki K, Nishijima T, Imanishi H, Ichimura K. Cell division and expansion growth during rose petal development. J Jpn Soc Hortic Sci. 2009;78(3):356-62.

26. Norikoshi R, Shibata T, Ichimura K. Cell division and expansion in petals during flower development and opening in Eustoma grandiflorum. Horticult J. 2016;85(2):154-60

27. Marowa P, Ding A, Kong Y. Expansins: roles in plant growth and potential applications in crop improvement. Plant Cell Rep. 2016;35(5):949-65.

28. Maurel C, Verdoucq L, Luu DT, Santoni V. Plant aquaporins: membrane channels with multiple integrated functions. Annu Rev Plant Biol. 2008;59: 595-624.

29. Kumar M, Lee SC, Kim JY, Kim SJ, Aye SS, Kim SR. Over-expression of dehydrin gene, OsDhn1, improves drought and salt stress tolerance through scavenging of reactive oxygen species in rice (Oryza sativa L.). J Plant Biol. 2014;57(6):383-93.

30. Yu H, Wan H, Cheng T, Jia W, Yang W, Pan H, Zhang Q. Comparative RNAseq analysis of transcriptome dynamics during petal development in Rosa chinensis. Sci Rep. 2017;7:43382

31. Ochiai M, Matsumoto S, Yamada K. Methyl jasmonate treatment promotes flower opening of cut Eustoma by inducing cell wall loosening proteins in petals. Postharvest Biol Technol. 2013;82(21):1-5.

32. He YM, Lin YJ, Zeng XC. Dynamic changes of jasmonic acid biosynthesis in rice florets during natural anthesis. Acta Agron Sin. 2012;38(10):1891-9.

33. Gallego-Bartolomé J, Blázquez MA. Molecular mechanism for the interaction between gibberellin and brassinosteroid signaling pathways in Arabidopsis. Proc Natl Acad Sci U S A. 2012;109(33):13446-51.

34. Li QF, Wang C, Jiang L, Li S, Sun SS, He JX. An interaction between BZR1 and DELLAs mediates direct signaling crosstalk between brassinosteroids and gibberellins in Arabidopsis. Sci Signal. 2012;5(244):ra72.

35. Devaiah BN, Madhuvanthi R, Karthikeyan AS, Raghothama KG. Phosphate starvation responses and gibberellic acid biosynthesis are regulated by the MYB62 transcription factor in Arabidopsis. Mol Plant. 2009;2(1):43-58

36. Olsen AN, Ernst HA, Leggio LL, Skriver K. NAC transcription factors: structurally distinct, functionally diverse. Trends Plant Sci. 2005;10(2):79-87.

37. Dai F, Zhang C, Jiang X, Kang M, Yin X, Lü P, Zhang X, Zheng Y, Gao J. RhNAC2 and RhEXPA4 are involved in the regulation of dehydration tolerance during the expansion of rose petals. Plant Physiol. 2012;160(4): 2064-82.

38. Nag A, King S, Jack T. miR319a targeting of TCP4 is critical for petal growth and development in Arabidopsis. Proc Natl Acad Sci U S A. 2009;106(52):22534-9.

39. Wang Y, Hu Y. Study of temperature influence on blooming of Osmanthus fragrans in Shanghai. In: Advances in Ornamental Horticulture of China: 2011: China Forestry Press; 2011. p. 768-71.

40. O'Donoghue EM, Somerfield SD, Heyes JA. Organization of cell walls in Sandersonia aurantiaca floral tissue. J Exp Bot. 2002;53(368):513-23.

41. Gookin TE, Hunter DA, Reid MS. Temporal analysis of alpha and betaexpansin expression during floral opening and senescence. Plant Sci. 2003; 164(5):769-81.

42. Harada T, Torii Y, Morita S, Onodera R, Hara Y, Yokoyama R, Nishitani K, Satoh S. Cloning, characterization, and expression of xyloglucan endotransglucosylase/hydrolase and expansin genes associated with petal growth and development during carnation flower opening. J Exp Bot. 2011; 62(2):815-23.
43. Singh AP, Dubey S, Lakhwani D, Pandey SP, Khan K, Dwivedi UN, Nath P, Sane AP. Differential expression of several xyloglucan endotransglucosylase/ hydrolase genes regulates flower opening and petal abscission in roses. Aob Plants 2013;5(1):plt030.

44. Fu C, Wang F, Liu W, Liu D, Li J, Zhu M, Liao Y, Liu Z, Huang H, Zeng X. Transcriptomic analysis reveals new insights into high-temperaturedependent glume-unclosing in an elite rice male sterile line. Front Plant Sci. 2017:8:112.

45. Reeves PH, Ellis CM, Ploense SE, Wu MF, Yadav V, Tholl D, Chételat A, Haupt I, Kennerley BJ, Hodgens C. A regulatory network for coordinated flower maturation. PLoS Genet. 2012;8(2):e1002506.

46. Hedden P, Thomas SG. Gibberellin biosynthesis and its regulation. Biochem J. 2012;444(1):11-25.

47. Razem FA, Baron K, Hill RD. Turning on gibberellin and abscisic acid signaling. Curr Opin Plant Biol. 2006;9(5):454-9.

48. Zhong K, Li X, Le X, Kong X, Zhang Z. MoDnm1 dynamin mediating peroxisomal and mitochondrial fission in complex with MoFis1 and MoMdv1 is important for development of functional appressorium in Magnaporthe oryzae. PLoS Pathog. 2016;12(8):e1005823.

49. Zhang C, Wang Y, Fu J, Bao Z, Zhao H. Transcriptomic analysis and carotenogenic gene expression related to petal coloration in Osmanthus fragrans 'Yanhong Gui'. Trees - Structure Function. 2016;30(4):1207-23.

50. Zhang C, Wang Y, Fu J, Dong L, Gao S, Du D. Transcriptomic analysis of cut tree peony with glucose supply using the RNA-Seq technique. Plant Cell Rep. 2014:33(1):111-29.

51. Grabherr MG, Haas BJ, Yassour M, Levin JZ, Thompson DA, Amit I, Adiconis X Fan L, Raychowdhury R, Zeng Q. Full-length transcriptome assembly from RNA-Seq data without a reference genome. Nat Biotechnol. 2011;29(7):644-52.

52. Pertea G, Huang X, Liang F, Antonescu V, Sultana R, Karamycheva S, Lee $Y$, White J, Cheung F, Parvizi B. TIGR gene indices clustering tools (TGICL): a software system for fast clustering of large EST datasets. Bioinformatics. 2003:19(5):651-2

53. Altschul SF, Gish W, Miller W, Myers EW, Lipman DJ. Basic local alignment search tool. J Mol Biol. 1990;215(3):403-10

54. Buchfink B, Xie C, Huson DH. Fast and sensitive protein alignment using DIAMOND. Nat Methods. 2015;12(1):59-60.

55. Quevillon E, Silventoinen V, Pillai S, Harte N, Mulder N, Apweiler R, Lopez R. InterProScan: protein domains identifier. Nucleic Acids Res. 2005;33:W116-20.

56. Conesa A, Götz S, García-Gómez JM, Terol J, Talón M, Robles M. Blast2GO: a universal tool for annotation, visualization and analysis in functional genomics research. Bioinformatics. 2005;21(18):3674-6.

57. Mortazavi A, W BA, M K, S L, W B. Mapping and quantifying mammalian transcriptomes by RNA-Seq. Nat Methods. 2008;5(7):621-8.

58. Audic S, Claverie JM. The significance of digital gene expression profiles Genome Res. 1997;7(10):986-95.

59. Zhang C, Fu J, Wang Y, Bao Z, Zhao H. Identification of suitable reference genes for gene expression normalization in the quantitative real-time PCR analysis of sweet osmanthus (Osmanthus fragrans Lour.). PLoS ONE. 2015; 10(8):e0136355.

\section{Publisher's Note}

Springer Nature remains neutral with regard to jurisdictional claims in published maps and institutional affiliations. 